\title{
Ultrafast circular polarization oscillations in spin-polarized vertical- cavity surface-emitting laser devices
}

\author{
N.C. Gerhardt*a, M. Li ${ }^{\mathrm{a}}$, H. Jaehme ${ }^{\mathrm{a}}$, H. Soldat ${ }^{\mathrm{a}}$, M.R. Hofmann ${ }^{\mathrm{a}}$ and T. Ackemann ${ }^{\mathrm{b}}$ \\ ${ }^{a}$ Photonics and Terahertz Technology, Ruhr-University Bochum, Universitaetsstr. 150, D-44780 \\ Bochum, Germany \\ ${ }^{\mathrm{b}}$ SUPA and Department of Physics, University of Strathclyde, Glasgow G4 0NG, Scotland, U.K.
}

\begin{abstract}
N.C. Gerhardt, M. Li, H. Jaehme, H. Soldat, M.R. Hofmann, and T. Ackemann. "Ultrafast circular polarization oscillations in spin-polarized vertical-cavity surface-emitting laser devices.” Physics and Simulation of Optoelectronic Devices XVIII, B. Witzigmann, F. Henneberger, Yasuhiko Arakawa, M. Osin'ski, (editors), Proc. SPIE 7597, 75970Q, 2010.

Copyright 2010 Society of Photo-Optical Instrumentation Engineers. One print or electronic copy may be made for personal use only. Systematic reproduction and distribution, duplication of any material in this paper for a fee or for commercial purposes, or modification of the content of the paper are prohibited.
\end{abstract}

http://dx.doi.org/ 10.1117/12.841606

\begin{abstract}
Spin-polarized lasers offer new encouraging possibilities for future devices. We investigate the polarization dynamics of electrically pumped vertical-cavity surface-emitting lasers after additional spin injection at room temperature. We find that the circular polarization degree exhibits faster dynamics than the emitted light. Moreover the experimental results demonstrate a strongly damped ultrafast circular polarization oscillation due to spin injection with an oscillation frequency of approximately $11 \mathrm{GHz}$ depending on the birefringence in the VCSEL device. We compare our experimental results with theoretical calculations based on rate-equations. This allows us to predict undamped long persisting ultrafast polarization oscillations, which reveal the potential of spin-VCSELs for ultrafast modulation applications.
\end{abstract}

Keywords: spin-VCSEL, spinoptoelectronic devices, spin injection, polarization dynamics, spin-flip model

\section{INTRODUCTION}

The development of semiconductor devices with new or enhanced functionalities on the basis of the spin of carriers is a field of very active research. In particular spin-controlled optoelectronic devices are of increasing interest due to the direct connection between the spin of the recombining carriers and the polarization of the emitted light ${ }^{1}$. In the past decade major breakthroughs on the way towards room temperature spin-optoelectronic devices have been achieved. Here most studies concentrate on investigating the electrical spin injection in light emitting diodes (LEDs) ${ }^{2-5}$. Polarization degrees up to $32 \%$ have been reported at room temperature with standard quantum well (QW) based LEDs ${ }^{4}$. Unfortunately such spin-LEDs typically require high external magnetic fields in the range of a few Tesla for efficient spin injection ${ }^{6}$. However recent progress based on ferromagnetic electrical spin-injection contacts with spontaneous perpendicular magnetization has enabled room-temperature spin-controlled light emitting diodes (spin-LEDs) operating even in remanence, i.e. without external magnetic fields ${ }^{5,7,8}$. This concept has also been transferred successfully to photodetectors: room-temperature spin-detectors for direct detection of polarized light using spin-filtering effects have been demonstrated ${ }^{9}$. The combination between efficient room-temperature spin emitters and detectors may enable optical transport of spin information over large distances for high-speed spin-communication technology in the future.

However, for such applications, a spin-LED does not fulfill the requirements concerning dynamics, polarization degrees and beam profile. With respect to these requirements, the combination of spin-injection with optical amplification in 
spin-lasers is much more promising ${ }^{10}$. In particular, spin vertical-cavity surface-emitting lasers (spin-VCSELs) have the potential even to outperform their conventional counterparts because of spin-specific advantages such as threshold reduction $^{11,12}$, ultrafast polarization switching capability ${ }^{13,14}$, and the ability for independent modulation of polarization and intensity. Most important, spin-VCSELs allow for direct polarization control and amplification of spin information due to their nonlinearity at threshold even at room temperature ${ }^{15}$. The realization of first electrically pumped spinVCSELs $^{16}$ though only operating at low temperatures up to now, demonstrates that spin-VCSELs have enormous potential to become realistic spin-optoelectronic devices.

*nils.gerhardt@rub.de; phone +49 23432 26514; fax +49 23432 14167; www.ptt.rub.de

Anyway, the development of an efficient room-temperature spin-laser that fully exploits the above mentioned potential requires a comprehensive understanding of the interaction between the spin induced polarization effects with birefringence and dichroism in these devices ${ }^{17,18}$. For instance, electrically pumped VCSEL structures usually suffer from linear predetermination of their polarization and from complex polarization dynamics caused by birefringence and dichroism in the device. For cw excitation, we were able to demonstrate, that these effects can be overcompensated by spin injection ${ }^{10,19}$. Therefore a control of the polarization of the laser emission in commercial cw electrical pumped devices even at room temperature is possible. However, since for applications in communication technology ultrafast modulation capability is of fundamental interest, an understanding of the stationary functionalities alone is not sufficient. Instead the complex interplay between spin and carrier dynamics, photon lifetime and polarization dynamics has to be taken into account ${ }^{20}$.

In this contribution we analyze the polarization dynamics of a commercial electrically pumped VCSEL after additional optical spin injection at room temperature. In the first part we present experimental results for the spin induced polarization dynamics. In the second part we compare our experimental results with theoretical calculations based on a rate-equation model in order to analyze the complex interplay between birefringence, dichroism and spin- and carrier relaxation after spin injection. The theoretical calculation enables us to predict conditions for long persisting polarization oscillations in spin-VCSELs.

\section{POLARIZATION DYNAMICS NEAR LASER THRESHOLD}

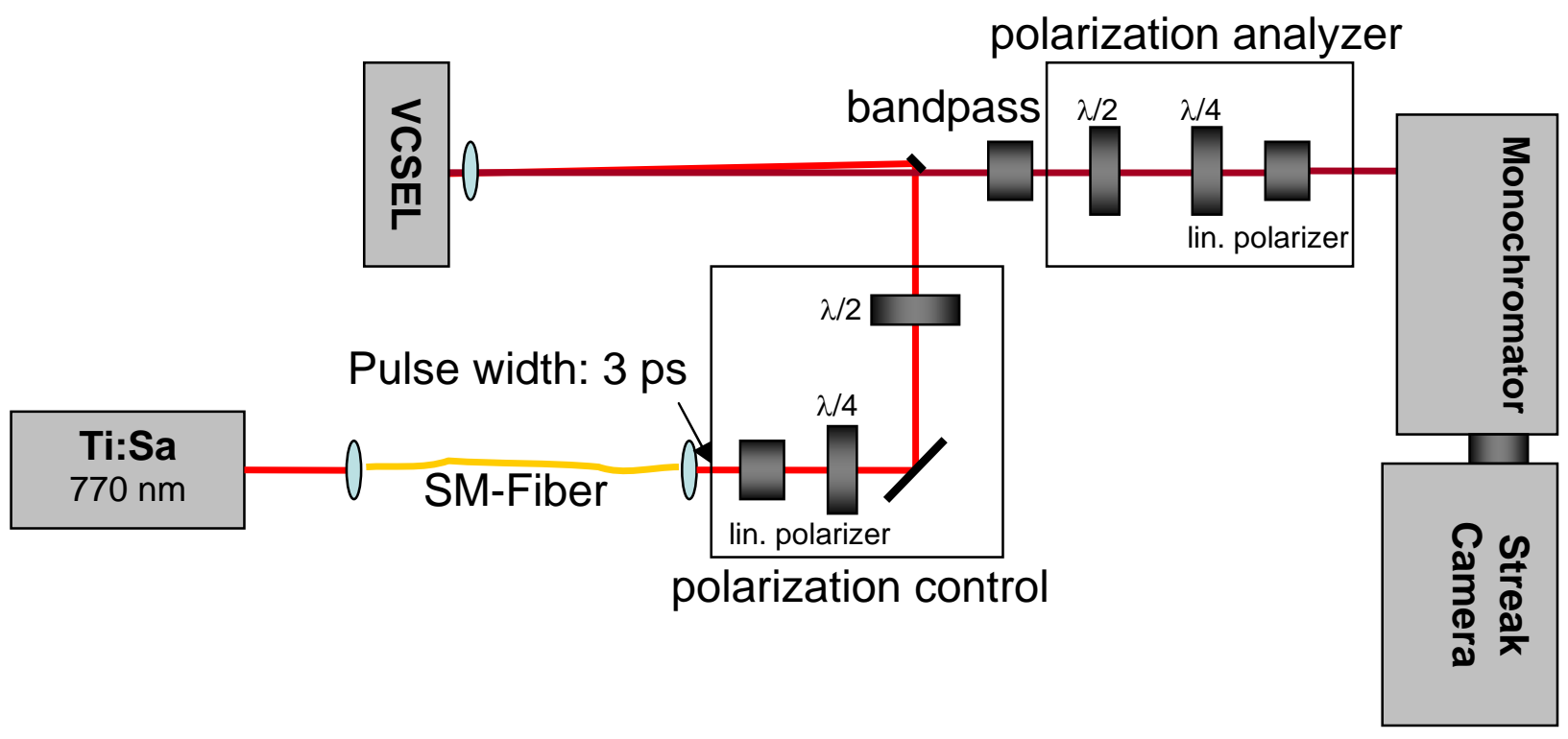


Figure 1: Experimental setup for the time-resolved polarization measurements of the VCSEL emission. The VCSEL will be pumped using a hybrid excitation technique. This technique combines continuous-wave electrical excitation with optical excitation by picosecond circular polarized laser pulses. The VCSEL emission will be analyzed by Stokes polarimeter followed by a ps streak-camera system.

Previous results about spin-induced effects in commercial electrically pumped VCSELs showed that the spin-polarized carriers generated by the pulsed circularly polarized optical excitation are able to determine the polarization of the VCSEL emission at room temperature ${ }^{10}$. But for cw operation, the spin control of the laser polarization works only for an electrical pump regime slightly below the electrical threshold. In this case, one of the spin band populations for the electrons reaches threshold earlier because of the anisotropic pumping. As a result the corresponding circularly polarized laser mode begins to build up, whereas the other mode stays suppressed. For steady state conditions, this effect will be negated for higher pump currents due to clamping of the band population at the laser threshold. Hence a pump region slightly below threshold is an optimum regime for cw operation of spin-VCSELs and is also an important start regime for the following time-resolved investigations.

\subsection{Experimental setup}

The experimental setup for the time-resolved polarization measurements of the VCSEL emission is presented in figure 1. The VCSEL structure will be pumped by a special hybrid excitation technique combining continuous-wave electrical excitation with optical excitation by picosecond circularly polarized laser pulses. For the optical excitation a modelocked Titanium:Sapphire laser at an excitation wavelength of $770 \mathrm{~nm}$ with a repetition rate of $75.5 \mathrm{MHz}$ has been used. The excitation wavelength corresponds to a reflection minimum of the microcavity. The pump beam is coupled through an optical isolator into a single-mode fiber to ensure a perfectly circular beam shape, because elliptical beam shapes cause a predetermined polarization state along the long axis of the ellipse ${ }^{21}$. The duration of the laser pulse after transmission through the single-mode fiber was 3ps. The pump beam passed a linear polarizer followed by a quarterwave plate in order to adjust the desired circular polarization for excitation. The polarization distortion induced by the following silver plated mirrors is compensated by a half-wave plate between the mirrors. The pump light is focused on the sample by the same lens, used for collimating the VCSEL emission. Surface reflected pump stray light is suppressed by an optical band pass filter in the detection path.

The VCSEL structure itself is mounted in a temperature stabilized sample holder and is pumped electrically under continuous wave conditions. The electrical injection introduces a statistical mixture of spin-up and spin-down electrons with an average spin polarization of zero. The pulsed circularly polarized optical excitation on the other hand introduces a comparatively small quantity of spin polarized electrons due to the optical selection rules ${ }^{1}$. With this hybrid pumping technique we achieve a variable anisotropic pumping for the spin subbands. The response of the VCSEL due to the hybrid excitation is then analyzed polarization-resolved, spectrally-resolved and time-resolved with a Stokes polarimeter followed by streak-camera system with 2ps time resolution.

\subsection{The sample}

We investigate a commercial proton-implanted VCSEL (type 8085-2010), fabricated by Emcore. The diameter of the emission window is $8 \mu \mathrm{m}$ ensuring a spatial single mode operation in the investigated current regime. Single mode operation is crucial for the following discussion and therefore has been verified experimentally using a high resolution spectrum analyzer in combination with beam profile measurements. The threshold current of the device is $4.7 \mathrm{~mA}$. At about $6.6 \mathrm{~mA}$ polarization switching (PS) can be observed in the VCSEL, i.e. the linear polarization state appearing at threshold is not stable but gives way to the orthogonal linear polarization state at the PS point. There are two different types of PS possible, here the VCSEL emission switches from the high to the low frequency mode with increasing current. More details about this sample type can be found in ${ }^{10,22-24}$.

\subsection{Experimental results and discussion}

The left side of figure 2 shows the time-resolved and spectrally-resolved total intensity after combined optical and electrical pumping $2 \%$ below the electrical threshold. The optical excitation was performed using a right circularly polarized laser pulse. The intensity dynamics shows the emission of a first strong pulse with 150ps width followed by several weaker satellite pulses after about 850ps and afterwards. The results reveal the typical relaxation oscillations after pulsed excitation. Because of the operation close to threshold, a nonzero background signal due to spontaneous emission is observed. The VCSEL emission spectrum is very narrow due to the single mode operation of the VCSEL, 
and only limited by the resolution of our spectrometer. The dynamics of the circular polarization degree (CPD), shown on the right part of figure 2, differs strongly from the intensity dynamics. In contrast to the intensity dynamics, the CPD exhibits a fast oscillation with a characteristic period shorter than 100ps all within the first intensity peak.

The comparison between total intensity and circular polarization degree as a function of time is plotted in figure 3 . The first peak of the CPD is right circularly polarized and therefore determined by the handedness of the excitation polarization. Then the CPD flips to a negative peak and the VCSEL emission turns left circularly polarized and so on. Thus the polarization dynamics shows an ultrafast oscillation with an oscillation frequency of approximately $11 \mathrm{GHz}$. Moreover, the CPD has much faster dynamics than the emitted intensity, which reveals the potential of spin-VCSELs for ultrafast modulation applications. Remarkably, this behavior is observable in absence of magnetic fields, hence differs from the Larmor precession in VCSELs reported by Hallstein et al. that was only observable under magnetic fields ${ }^{25}$.
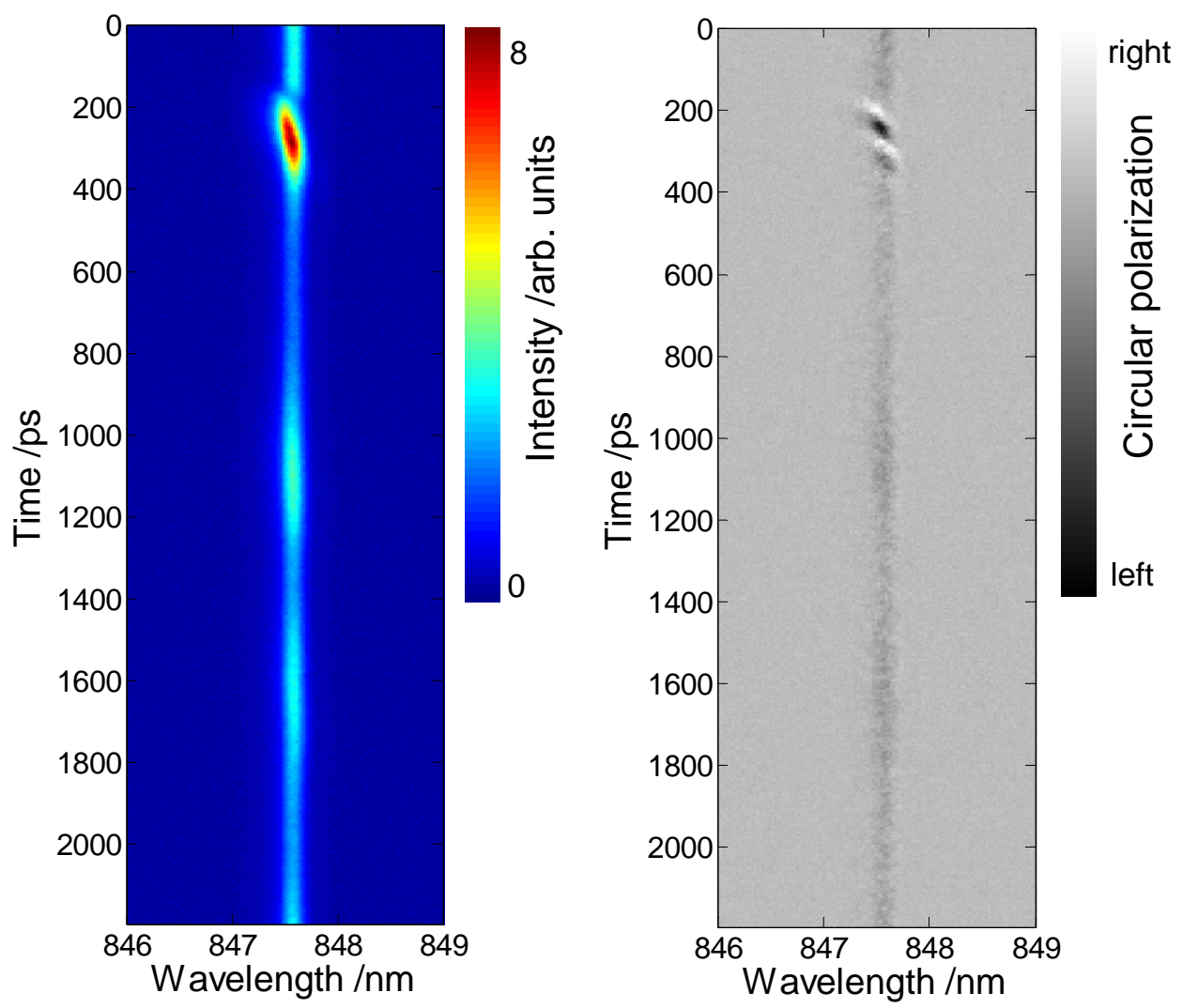

Figure 2. Time-resolved and spectral-resolved response of the VCSEL after combined spin-polarized optical and unpolarized electrical pumping $2 \%$ below the electrical threshold. The left part presents the total intensity whereas the right part shows the circular polarization in greyscale (white: right circular, black left circular polarization). The optical pump pulse had a right circular polarization state. 


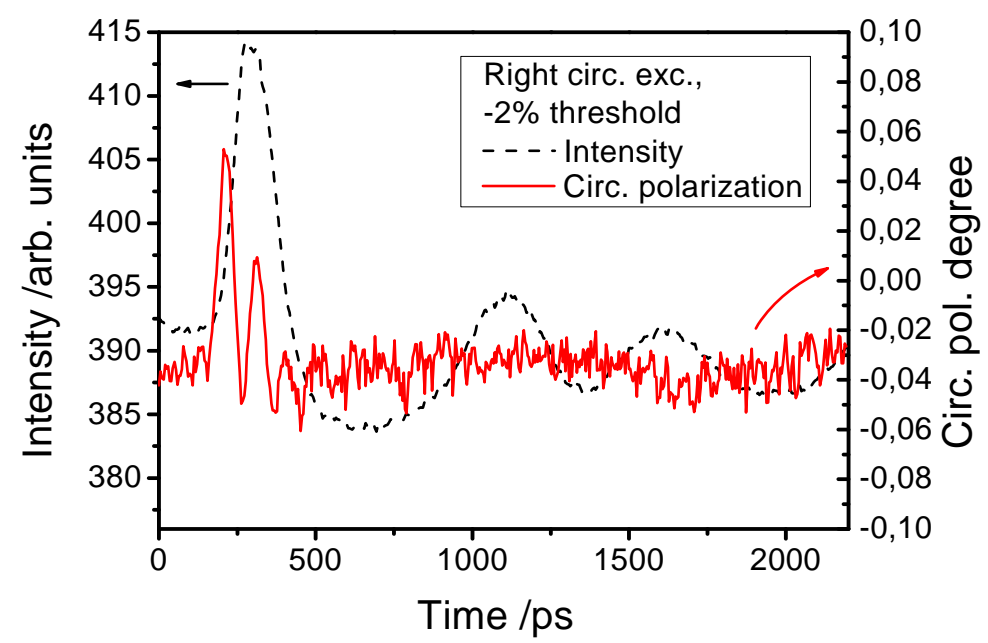

Figure 3. Time-resolved intensity and polarization dynamics for the spectrally integrated VCSEL emission after hybrid pumping $2 \%$ below the electrical threshold. The results show circular polarization oscillations with an oscillation frequency of approximately $11 \mathrm{GHz}$.

Unfortunately, the polarization oscillations can be observed only during the first intensity pulse. Therefore, the oscillations are strongly damped, which, on the other side, could be an important drawback for practical applications.

The observed oscillations are a consequence of the complex interplay of the spin dynamics with birefringence and dichroism in the VCSEL cavity. Under right circular polarized excitation, the electrons are mostly excited to the spin up subband, due to the optical selection rules. They are able to recombine with holes from the valence band, which are considered to be spin unpolarized due to the short hole-spin relaxation time at room temperature. Therefore the observed circular polarization degree is connected to the spin dynamics of the electrons in the conduction band only. As discussed earlier, the hybrid pumping regime leads to a small anisotropic pumping of the electron-spin subbands, and the spin up subband reaches threshold earlier. As a result, a right circularly polarized laser mode will be emitted. However, circular polarization modes are typically not stable in this regime in contrast to linear polarization modes. The birefringence in the cavity couples the circular polarization components with different helicity and leads hence to an oscillation between them. During this oscillation the spin populations of the spin subbands will be depleted in an anisotropic way feeding back into the polarizations dynamics. Additionally the dynamic unbalance of the spin subbands will be reduced permanently by the fast spin relaxation at room temperature and probably due to linear and nonlinear dichroism in the cavity.

Such oscillations for the circular polarization degree could be observed indirectly before, first of all in the noise spectrum of linearly polarized states ${ }^{26}$ and secondly in the transients of a polarization switching between linearly polarized states ${ }^{27}$. Here we demonstrate them directly as large amplitude oscillations between circular polarized states. The oscillation frequency is usually given by the linear birefringence and typically small corrections due to nonlinear spin effects ${ }^{26,28}$. The birefringence of electrically pumped VCSELs consists of a deterministic part due to the electro-optical effect and an unintentional part due to strain induced by processing or even mounting ${ }^{18}$. The birefringence due to strain can be widely tuned, which might be an easy and promising approach to use the polarization oscillations in spin-VCSELs for ultrafast modulation applications. But a remaining drawback on this way is still the strong damping of the oscillations. In order to understand the reasons for this strong damping and to find a way to reduce this damping, we analyze the polarization oscillations by means of a rate-equation model in the next section. 


\section{SPIN-FLIP MODEL}

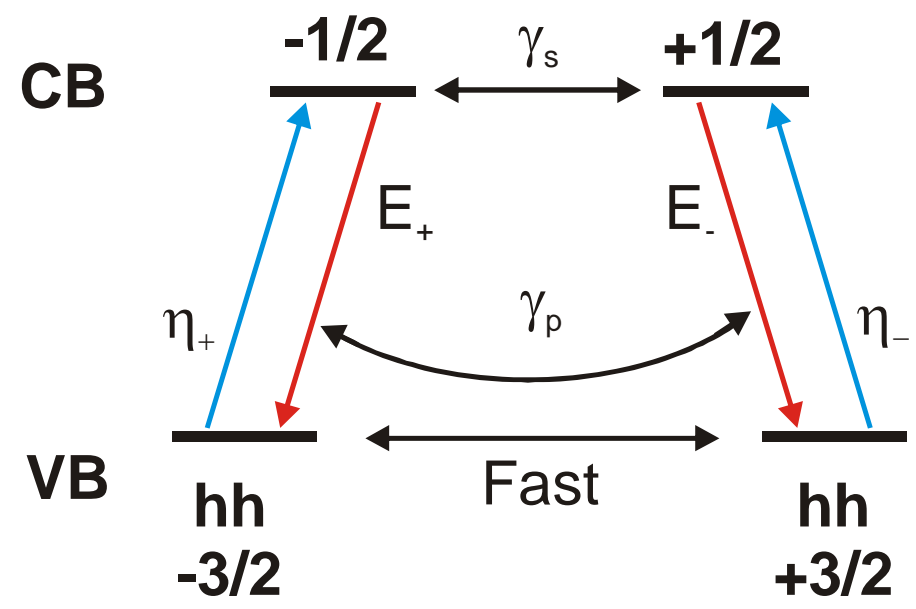

Figure 4. The simplified 4 level system used in the spin flip model for the VCSEL emission. The model includes recombination between the electron states in the conduction band (CB) and the heavy hole (hh) states in the valence band (VB) only.

\subsection{Model description}

In this section we compare our experimental results with numerical simulations based on a spin flip model (SFM). The model has been developed by Gahl et al. ${ }^{29}$ and describes the slowly varying complex amplitude of the two circularly polarized fields $E_{ \pm}$with the corresponding intensities $I_{ \pm}=\left|E_{ \pm}\right|^{2}$, the normalised carrier density $\mathrm{N}$, and the imbalance of the two spin polarisations in a coupled rate equation system:

$$
\begin{aligned}
& \dot{E}_{ \pm}=\kappa(1+i \alpha)\left(N \pm m_{z}-1\right) E_{ \pm}-\left(\gamma_{a}+i \gamma_{p}\right) E_{\mp}+\sqrt{\beta \gamma\left(N \pm m_{z}\right)} \xi_{ \pm}, \\
& \dot{N}=\gamma\left[\eta_{+}+\eta_{-}-\left(1+I_{+}+I_{-}\right) N-\left(I_{+}-I_{-}\right) m_{z}\right], \\
& \dot{m}_{z}=\gamma\left(\eta_{+}-\eta_{-}\right)-\left[\gamma_{s}+\gamma\left(I_{+}+I_{-}\right)\right] m_{z}-\gamma\left(I_{+}-I_{-}\right) N
\end{aligned}
$$

$\eta_{+}\left(\eta_{-}\right)$is the time dependent optical pump of the $E_{+}\left(E_{-}\right)$component, $\gamma$ the electron density decay rate, and $m_{z}$ the electron magnetization with the decay rate $\gamma_{s}$ due to spin flip processes. $\kappa$ is the cavity decay rate, $\alpha$ the linewidth enhancement factor and $\beta$ the spontaneous emission factor. $\xi_{ \pm}$are complex Gaussian white noise sources of zero mean value and $\delta$-correlated in time, $\gamma_{p}$ is the linear birefringence, and $\gamma_{a}$ the gain anisotropy.

The description of the semiconductor in this model is simplified to a 4 level system as displayed in figure 4 , including only the heavy hole and electron states with their two opposite spin orientations. In this model the spin populations in the conduction band are coupled by the spin relaxation rate $\gamma_{s}$, whereas the spin relaxation time for the hole-spin is assumed to be instantaneous. The following design dependent parameters were used for the calculations if not stated otherwise: $\gamma=25 n s^{-1}, \gamma_{s}=100 n s^{-1}, \kappa=250 n s^{-1}, \alpha=2, \gamma_{p}=20 G H z, \gamma_{a}=-1.65 G H z, \beta=10^{-4}$. 


\subsection{Theoretical results and discussion}

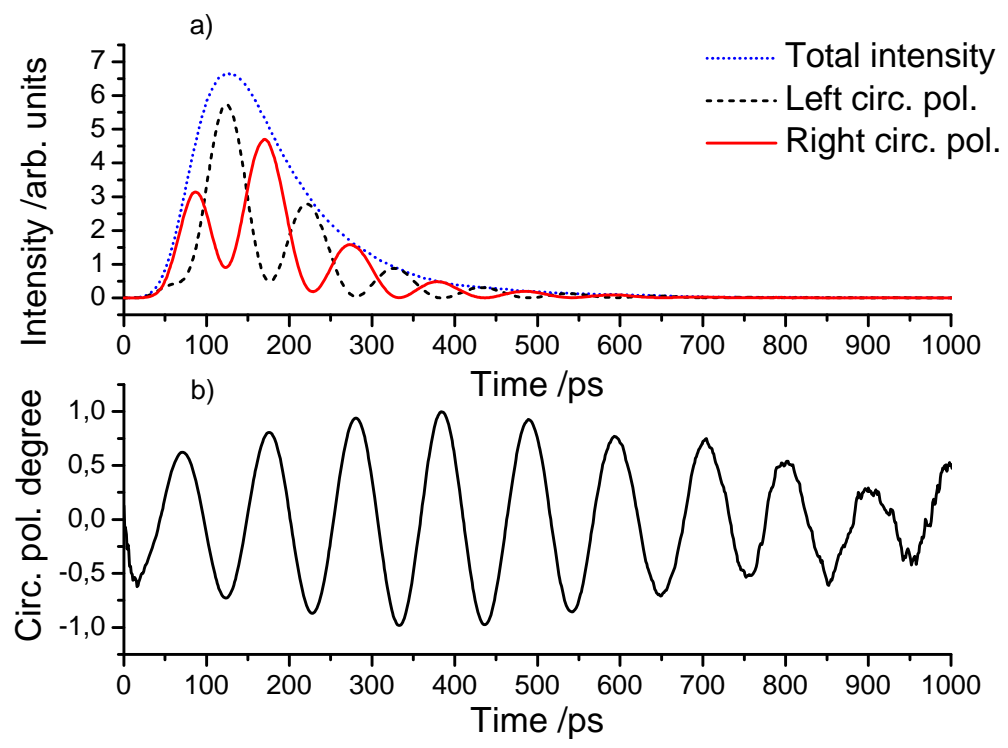

Figure 5. Theoretical calculations by the spin-flip model for the intensity dynamics (a) and the circular polarization dynamics (b) after hybrid excitation $2 \%$ below threshold.

Figure 5 shows a typical result from the rate-equation model for a hybrid excitation regime slightly below electrical threshold. As observed experimentally in section 2 the anisotropic pumping leads to an unbalanced spin band population. The spin up band population reaches threshold earlier and a short emission takes place on the right circularly polarized laser mode. Then the CPD flips to a negative peak and so on. Therefore the calculations show fast circular polarization oscillations comparable to the experimental results with a clear damping behavior. The damping is not as strong as in the experimental results, because obviously the lack of light after the first pulse avoids an adequate measurement of the circular polarization degree in the experiment. Nevertheless the oscillation was completely suppressed during the second pulse, i.e. after 850 ps (see Fig.3). In the theoretical results the oscillations are clearly visible even after the first laser pulse but also show a clear damping behavior over a period of one nanosecond (ns) after optical excitation.

With this theoretical model which is able to reproduce the observed experimental behavior, we have now the possibility to investigate the origin of the strong oscillation damping. One important parameter could be the spin relaxation time, which cannot be changed easily in a realistic device. One possible approach is to use (110)-GaAs substrates leading to a longer spin relaxation time. Though the first optically pumped VCSELs on (110)-substrate have been realized recently ${ }^{14}$, it is still a big challenge to transfer this concept to electrically pumped VCSELs in the future. Besides the spin relaxation time another important parameter should be the effective dichroism, defined as the sum of the gain and loss anisotropies in the VCSEL structure. In contrast to the spin relaxation time, the effective dichroism can be affected easily even in commercial devices. In the case of high to low polarization switching, for example, as observed in our sample, the effective dichroism reaches a minimum at the PS point ${ }^{26,30,31}$. 


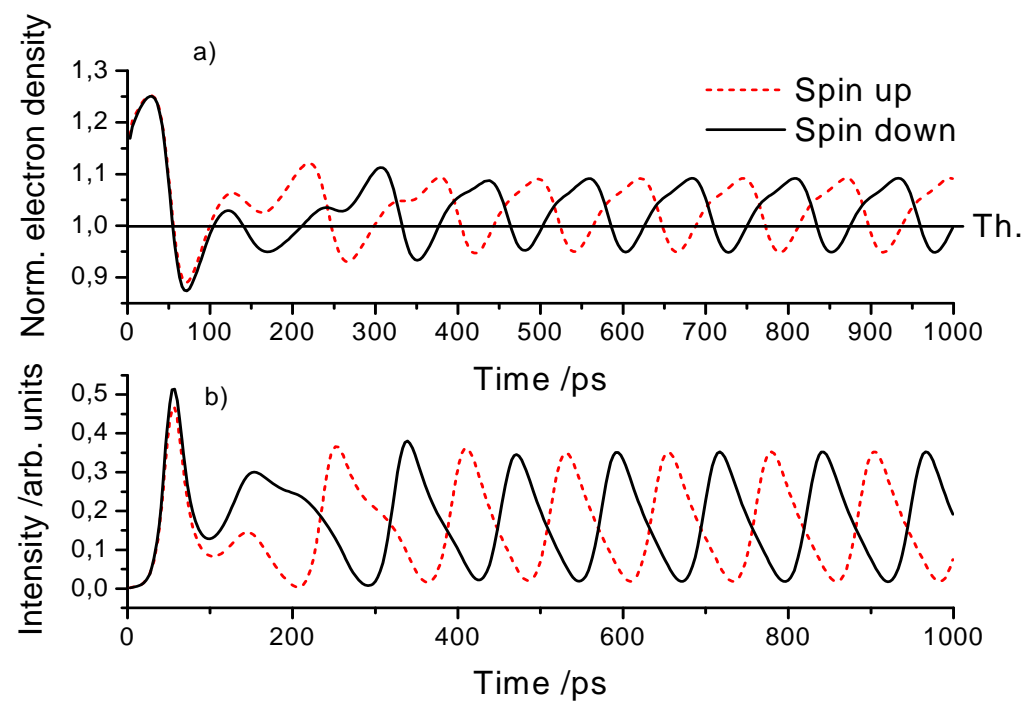

Figure 6. Theoretical calculations for the spinband population (a) and the circularly polarized intensities (b) at the polarization switching point of the spin-VCSEL device. The model predicts ultra long persisting CPD oscillations.

Consequently figure 6 shows theoretical calculations for the polarization (b) and population dynamics (a) at this interesting polarization switching point. The results clearly demonstrate that the undesired damping of the polarization oscillations can be reduced efficiently and long persisting oscillations of the CPD over time periods much longer than the spin relaxation time should be possible in this special regime. This reveals the potential of spin-controlled VCSELs for ultrafast polarization modulation with frequencies much higher than possible with the conventional intensity modulation of VCSELs.

The circular polarization oscillations at the polarization switching point are presented in figure 7 as a function of current and for three different values of the birefringence. The current dependence of the polarization oscillations illustrates that the long persisting oscillations are actually restricted to a small current regime around the polarization switching point, where the effective dichroism is very small in our device. This indicates that the dichroism is an important parameter for the oscillation damping. Moreover the width of the interesting region as well as the oscillation frequency can be tuned by the birefringence. This supports the above mentioned idea that the control of the birefringence in VCSELs for example by strain is a very promising approach for future spin-VCSEL devices.

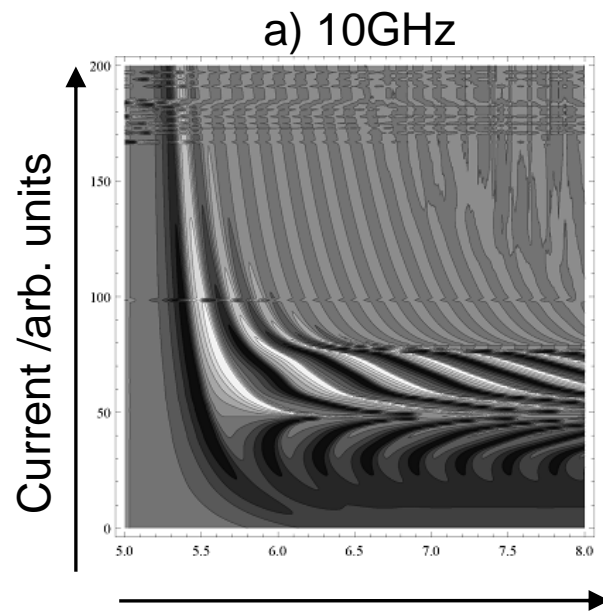

Time /ns b) $20 \mathrm{GHz}$

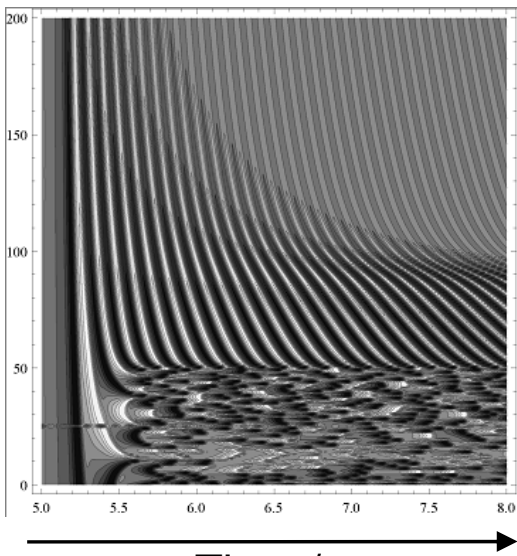

Time /ns c) $30 \mathrm{GHz}$

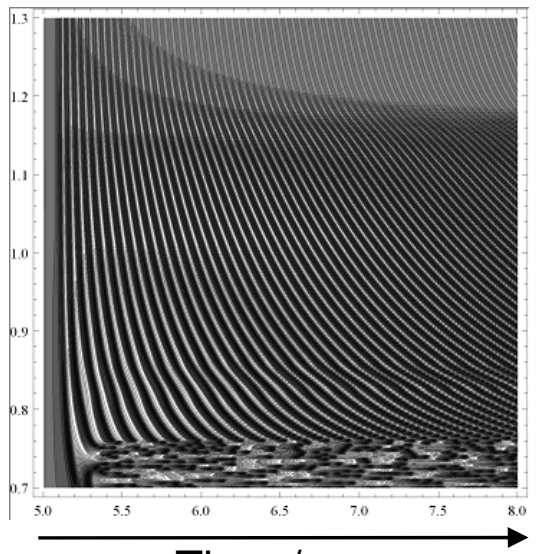

Time /ns 
Figure 7. Theoretical calculations for the dynamics of the CPD near the polarization switching point. The CPD is plotted in circular polarization in greyscale for three different values for the birefringence.

\section{CONCLUSIONS}

In summary, we have investigated the polarization dynamics of an electrically pumped vertical-cavity surface-emitting laser after additional spin injection at room temperature. We could demonstrate experimentally that the circular polarization degree exhibits faster dynamics than the emitted light. The experimental results showed a strongly damped ultrafast circular polarization oscillation due to spin injection with an oscillation frequency of approximately $11 \mathrm{GHz}$ for hybrid pumping near the electrical threshold. We have compared our experimental results with theoretical calculations based on a spin-flip model which predicts undamped long persisting ultrafast polarizations oscillations at room temperature for a pump regime near the polarization switching point. These results demonstrate clearly the potential of future spin-controlled VCSELs for ultrafast modulation applications. The next important step on the way to spinVCSELs is the experimental verification of this behavior in commercial VCSEL devices.

\section{ACKNOWLEDGEMENTS}

The authors thank the Deutsche Forschungsgesellschaft (DFG) for support within the Sonderforschungsbereich 491.

\section{REFERENCES}

[1] Meier, F. and Zakharchenya, B.P., [Optical Orientation], Elsevier Science Publisher B.V.,11-73 (1984).

[2] Zhu, H.J., Ramsteiner, M., Kostial, H., Wassermeier, M., Schönherr, H.-P., and Ploog, K.H., "Room-Temperature Spin Injection from Fe into GaAs ,“ Phys. Rev. Lett. 87, 016601 (2001).

[3] Adelmann, C., Lou, X., Strand, J., Palmstrom, C.J., Crowell, P.A., "Spin injection and relaxation in ferromagnetsemiconductor heterostructures, ” Phys. Rev. B- B71, 121301 (2005)

[4] Jiang, X., Wang, R., Shelby, R.M., MacFarlane, R.M., Bank, S.R., Harris, J.S.,and Parkin, S.S.P., "Highly spinpolarized room-temperature tunnel injector for semiconductor spintronics using MgO(100), " Phys. Rev. Lett. 94, 056601 (2005).

[5] Gerhardt, N.C., Hövel, S., Brenner, C., Hofmann, M.R., Lo, F.-Y., Reuter, D., Wieck, A.D., Schuster E. and Keune, W., "Electron spin injection into GaAs from ferromagnetic contacts in remanence, " Appl. Phys. Lett. 87, 032502 (2005). [6] Hanbicki, A.T., Jonker, B.T.Itskos, G., Kioseoglou, G., Petrou, A.,”Efficient electrical spin injection from magnetic metal/tunnel barrier contact into a semiconductor,” Appl. Phys. Lett. 80, 1240 (2002).

[7] Hövel, S., Gerhardt, N.C., Hofmann, M.R., Lo, F.-Y., Ludwig, A., Reuter, D., Wieck, A.D., Schuster E., Wende, H., Keune, W., Petracic, O. and Westerholt, K., "Room temperature electrical spin injection in remanence, " Appl. Phys. Lett. 93, 021117 (2008).

[8] Sinsarp, A., Manago, T., Takano, F., and Akinaga, H., "Electrical Spin Injection from Out-of-Plane Magnetized FePt/MgO Tunneling Junction into GaAs at Room Temperature," Jap. J. Appl. Phys. 46, L4 (2007)

[9] Hövel, S., Gerhardt, N.C., Hofmann, M.R., Lo, F.-Y., Reuter, D., Wieck, A.D., Schuster E., Keune, W., Wende, H., Petracic, O. and Westerholt, K., "Electrical detection of photoinduced spins both at room temperature and in remanence," Appl. Phys. Lett. 92, 242102 (2008).

[10] Hövel, S., Bischoff, A., Gerhardt, N.C., Hofmann, M.R., Ackemann, T., Kroner, A. and Michalzik, R., "Optical spin manipulation of electrically pumped vertical-cavity surface-emitting lasers," Appl. Phys. Lett. 92, 041118 (2008).

[11] Oestreich, M., Hübner, J., Hägele, D., Bender, M., Gerhardt, N.C., Hofmann, M.R., Rühle, W.W., Kalt, H., Hartmann, T., Klar, P., Heimbrodt, W. and Stolz, W., "Spin injection, spin transport and spin coherence," Advance in solid state physics 41, 173 (2001).

[12] Gerhardt, N.C., Hövel, S., Hofmann, M.R., Yang, J., Reuter, D and Wieck, A.D., "Enhancement of spin information with vertical cavity surface emitting lasers" Electron. Lett., 42, 88 (2006).

[13] Basu, D., Saha, D. and Bhattacharya, P., "Optical Polarization Modulation and Gain Anisotropy in an Electrically Injected Spin Laser," Phys. Rev. Lett. 102, 093904 (2009).

[14] Ikeda, K., Fujimoto, T., Fujino, H., Katayama, T., Koh, S and Kawaguchi, H., "Switching of Lasing Circular Polarizations in a (110)-VCSEL," IEEE Photon. Tech. Lett. 21, 1350 (2009).

[15] Ando, H., Sogawa, T. and Gotoh, H., "Photon-spin controlled lasing oscillation in surface-emitting lasers," Appl. Phys. Lett. 73, 566 (1998). 
[16] Holub, M., Shin, J., Saha, D. and Bhattacharya, P., "Electrical Spin injection and Threshold Reduction in a Semiconductor Laser," Phys. Rev. Lett. 98, 146603 (2007).

[17] San Miguel, M., Feng, Q. and Moloney, J.V., "Light-polarization dynamics in surface-emitting semiconductor lasers," Phys. Rev. A 52, 1728 (1995).

[18] Jansen van Doorn, A.K., van Exter, M.P. and Woerdman, J.P., "Elasto-optic anisotropy and polarization orientation of vertical-cavity surface-emitting semiconductor lasers," Appl. Phys. Lett. 69, 1041 (1996).

[19] Hövel, S.; Gerhardt, N. C.; Hofmann, M. R.; Lo, F.-Y.; Reuter, D.; Wieck, A. D.; Schuster, E.; Wende, H.; and Keune, W., "Spin-controlled optoelectronic devices," physica status solidi (c) 16, 436 (2009).

[20] Gothgen, Ch., Oszwaldowski, R., Petrou A., and Zutic I., "Analytic model of spin-polarized semiconductor lasers", Appl. Phys. Lett. 93, 042513 (2008).

[21] Choquette, K.D., Richie, D.A. and Leibenguth, R.E., "Temperature dependence of gain-guide vertical-cavity surface emitting laser polarization," Appl. Phys. Lett. 64, 2062 (1994).

[22] Ackemann, T. and Sondermann, M., "Characteristics of polarization switching from the low to the high frequency mode in vertical-cavity surface-emitting lasers," Appl. Phys. Lett. 78, 3574 (2001).

[23] Sondermann, M., Weinkath, M. and Ackemann, T., "Polarization Switching to the Gain Disfavored Mode in Vertical-cavity Surface-emitting Lasers, " IEEE J. Quantum Electron. 40, 97 (2004).

[24] Sondermann, M., Ackemann, T., Mulet, J., Balle, S. and Panajotov, K., "Experimental and theoretical investigations on elliptically polarised dynamical transition states in polarisation switching of vertical-cavity surface-emitting lasers," Opt. Commun. 235, 421 (2004).

[25] Hallstein, S., Berger, J.D., Hilpert, M., Schneider, H.C., Rühle, W.W., Jahnke, F., Koch, S.W. Gibbs, H.M., Khitrova, G. and Oestreich, M., "Manifestation of coherent spin precession in stimulated semiconductor emission dynamics ," Phys. Rev. B 56, R7076 (1997).

[26] van Exter, M.P., Willemsen, M.B. and Woerdman, J.P., "Polarization fluctuations in vertical-cavity semiconductor lasers," Phys. Rev. A 58, 4191 (1998).

[27] Willemsen, M.B., van Exter, M.P.and Woerdman, J.P., "Anatomy of a Polarization Switch of a Vertical-Cavity Semiconductor Laser," Phys. Rev. Lett. 84, 4337 (1999).

[28] Hofmann, H.F. and Hess, O., "Quantum noise and polarization fluctuations in vertical-cavity surface-emitting lasers," Phys. Rev. A 56, 868 (1996).

[29] Gahl, A., Balle, S. and San Miguel, M., "Polarization dynamics of Optically pumped VCESLs," IEEE J. Quan. Electron. 35, 342 (1999).

[30] Ackemann, T. and Sondermann, M., "Characteristics of polarization switching in vertical-cavity surface-emitting lasers," Proc. SPIE 4286, 44-54 (2001).

[31] van Exter, M.P., Willemsen, M.B. and Woerdman, J.P., " Polarization modal noise and dichroism in vertical-cavity semiconductor lasers," Appl. Phys. Lett. 74, 2274 (1999). 